\title{
Energy-Harvesting Materials
}

\author{
David L. Andrews* \\ School of Chemical Sciences, University of East Anglia, Norwich NR4 7TJ, U.K.
}

\begin{abstract}
It is shown how key features of natural photosynthesis can be emulated in novel materials based on photoactive multichromophore arrays and crystals. A major consideration in the design of such systems is the means of channeling electronic excitation from sites of light absorption to centers where it is stored or released. Storage is often achieved by driving charge separation or, for the longer term, a more complex chemical reaction whilst rapid release is commonly associated with frequency up-converted emission. In each case channeling to the conversion site generally entails a multi-step energy transfer mechanism whose efficiency is determined by the arrangement and electronic properties of the array chromophores or ions, guided in the more complex systems by a spectroscopic gradient that promotes overall directionality. The functional cascade molecules known as photoactive dendrimers are exemplars of this approach. The latest developments involve new mechanisms for concerted excitation transfer in multichromophore systems, leading towards the tailoring and exploitation of optical nonlinearities for high intensity energy pooling applications.
\end{abstract}

Keywords: Energy harvesting, solar energy, photosynthesis, dendrimers, nonlinear optics, photoactive materials, optoelectronics, photonics

\section{INTRODUCTION}

In the pursuit of greater control and economy in the global utilisation of energy, the quest for increasingly efficient mimics of natural photosynthesis has driven research down a multitude of paths. Many attempts to match the high efficiency of plants in harvesting solar power have been based on systems operating on considerably different principles - which is not surprising in view of the extreme chemical complexity of photobiological systems. However, as the detailed molecular structure and chromophore layout of the photosynthetic apparatus in a variety of organisms have begun to emerge, new avenues of research have recently led to significant advances in the modeling, synthesis and operation of more truly biomimetic energy harvesting materials. Whilst a host of possible energy storage mechanisms can be envisaged, key to the viability of any such system is the efficient channeling of electronic excitation to the storage centers. In this paper the principles that determine that transfer efficiency are examined, and exemplified with reference to natural photosynthetic systems and also photoactive dendrimers. It is also shown how the science of such materials links and is informed by developments in rare-earth based optoelectronic systems. Looking ahead, emerging research suggests that utilizing the nonlinear optical response of nanoscale materials will offer new and promising avenues for energy harvesting.

\section{BIOLOGICAL SYSTEMS}

It is instructive to begin with a brief overview of the basic energy harvesting strategy deployed by Nature. In photobiological systems, such harvesting is not primarily accomplished for the storage of energy as such, but as a means for the construction of sugars. The photosynthesis of each elementary carbohydrate unit $\left(\mathrm{CH}_{2} \mathrm{O}\right)$ in plants is achieved by two coupled redox reactions; one is the reduction of carbon dioxide, the other is the oxidation of water. Each of these requires the energy of four photons for its completion; thus, the energy of eight photons is consumed in the production of each carbohydrate unit. It is because real chemical change takes place that large numbers of (visible range) photons are required; singly, such photons lack the energy necessary to break chemical bonds. The purpose of man-made energy harvesting materials is not usually chemical synthesis; however both artificial and natural systems share a common need to capture and efficiently channel optical energy to a suitable site of repository, and it is here that crucial lessons can be learned from the structure and operation of biosystems.

\footnotetext{
*david.andrews@physics.org; phone +44 1603 592014; fax +44 1603 592003; http:// www.uea.ac.uk/ c051; School of Chemical Sciences, University of East Anglia, Norwich NR4 7TJ, U.K.
} 
Natural photosystems generally comprise several non-covalently bound pigment-protein complexes. In each complex the major protein component represents a superstructure within which the pigment molecules are held. In the visible region, the optical properties of such complexes are largely determined by the pigments, though the detailed spectral signatures of the latter are strongly influenced by their electronic surroundings. Purple photosynthetic bacteria have light-harvesting systems whose mechanistic and structural properties have been very thoroughly characterized by

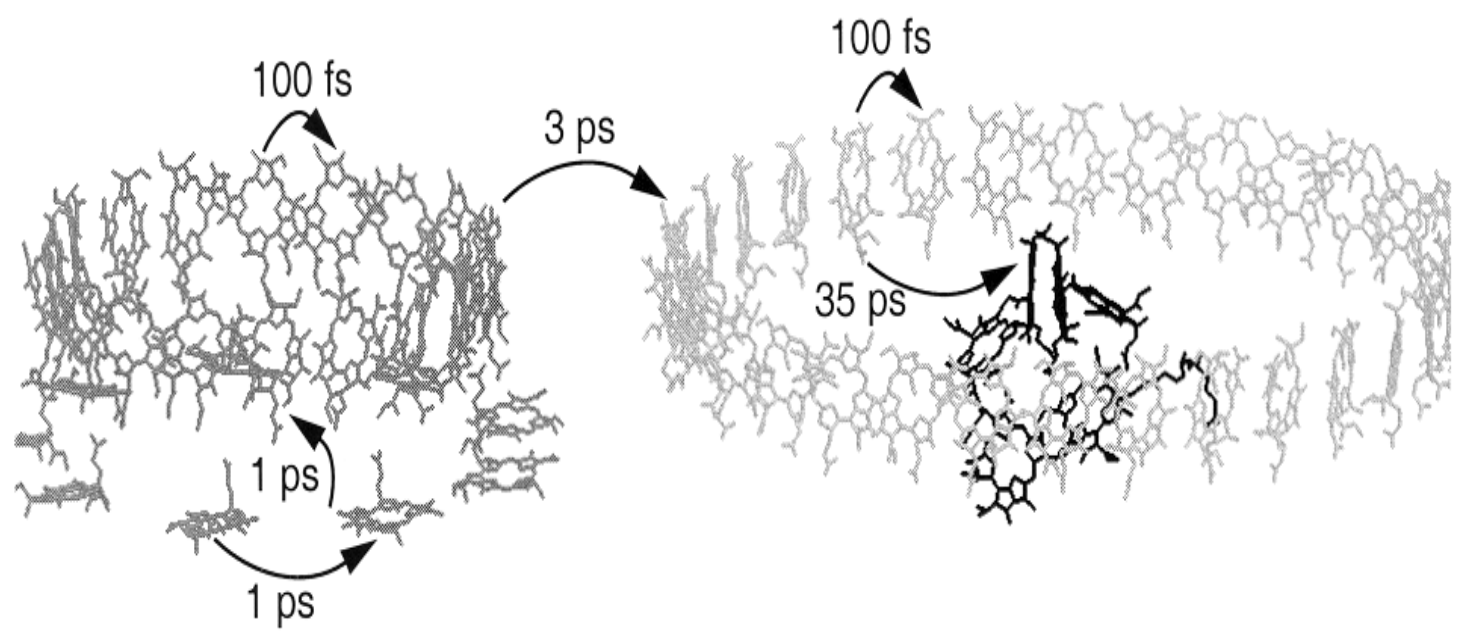

Fig. 1: Key energy transfer processes in the light-harvesting complexes of purple photobacteria (adapted from Ref. 1)

exploiting advanced ultrashort pulsed laser instrumentation, and X-ray protein crystallography. The following is a highly simplified summary of the structure and photophysics operative for most purple photobacteria; Van Grondelle and Somsen [1] and also Schulten [2,3] give details in recent reviews. Here the photosynthetic unit comprises pigmentprotein complexes within which the key light-harvesting pigment is a form of chlorophyll known as bacteriochlorophyll. Two of the light-harvesting complexes, designated LH1 and LH2, form coplanar ring structures as schematically depicted by Fig. 1; in reality there are many such LH2 rings around each LH1. In each circular aggregate, the bacteriochlorophyll pigments are in the form of dimers whose wavelength of maximum absorption is significantly modified by their protein environment. To simplify the terminology the bacteriochlorophyll dimer with an absorption maximum at $800 \mathrm{~nm}$, for example, is commonly referred to as B800. Thus LH2, on the left of Fig. 1, comprises both B850 (above) and B800 rings (below) whilst LH1, on the right, has a B880 ring. Together, these complexes deliver light energy to the reaction center (RC, shown at the center of LH1) where the photosynthetic chemistry ensues. Other ancillary carotenoid pigments absorb in other spectral regions, assisting the efficient capture of solar energy.

The first striking feature is the very rapid transfer of energy both within and between the light-harvesting complexes. Indeed, it was only as the technology of laser pulsing drove the temporal resolution down to below the femtosecond level that the true timescales for these ultrafast processes could be accurately determined. In these light-harvesting arrays, the operation of a spectroscopic gradient ensures that multi-step energy migration is not simply a random walk; each transfer from one bacteriochlorophyll species to another absorbing at a longer wavelength signifies a small but significant loss of energy (ultimately manifest as heat). For any one transfer step, a key factor in determining its efficiency is the extent of overlap between the emission spectrum of the species from which energy departs (which in this connection assumes the role of donor - let us call it $A$ ) and the absorption spectrum of the species to which energy transfers (the acceptor, $B$ ). In any subsequent migration, where the erstwhile acceptor $B$ plays the role of donor, any possible back-transfer to $A$ is largely inhibited by the much poorer overlap between the emission spectrum of $B$ and the absorption spectrum of $A$. For this reason the transfer of excitation energy towards the reaction center is favoured, a feature often referred to as channeling or funneling; the same principle also assists the one-way transfer of energy from carotenoids to chlorophyll species. To develop a more complete understanding of the factors that determine transfer efficiency, however, we need to look more closely at the underlying photophysics. 


\section{TRANSFER OF EXCITATION}

First, it can be recognized that more than one kind of excitation transfer is to be expected. Even the above introduction to the principle of a spectroscopic gradient strongly suggests the possibility of a difference in behavior when the donor and acceptor species are identical, as for example when we consider excitation transfer within one of the lightharvesting ring structures. Moreover the possibility of close proximity between donor and (neighboring) acceptor within a ring structure clearly allows for much stronger coupling than could occur between well separated chromophores. In the following, the main principle governing the transfer of excitation between environmentally different, well-separated chromophores is first outlined, and the modified behavior observed in the identical-neighbor case is then identified.

The primary mechanism for energy transfer in natural and synthetic harvesting structures goes by a variety of names, each signifying a different aspect of the coupling. It is incoherent energy transfer, often called radiationless, or resonance energy transfer (RET), [4-6] also often designated FRET in connection with the ensuing fluorescence. In honour of the instigator of the first theory, which was developed using perturbation theory based on dipole-dipole interaction between the excited donor and unexcited acceptor (the two dipoles involved being transition dipoles for the decay of the donor and the excitation of the acceptor), it is also known as Förster energy transfer. [7] The physical condition for this form of energy transfer is essentially weak coupling between the donor and acceptor species. In the modern theory based on quantum electrodynamics (QED) the coupling between the donor and acceptor transitions is calculated in terms of mediation by a photon that has to be conceptualized as a virtual quantity - its involvement can only be inferred, and the energy transfer is for all practical purposes radiationless. [8] The QED theory in fact embraces both radiationless (Förster) and long-range radiative energy transfer - the results are valid over the whole span of distances ranging from those which characterize molecular structure (nanometers) up to laboratory-scale distances and beyond [8-11]. Here, restricting consideration to donor-acceptor separations $R$ substantially smaller than the wavelengths of visible radiation, the theory gives the following expression for the rate of pairwise energy transfer $w$, applicable for systems where the host structure for the donor and acceptor has refractive index $n$ :

$$
w=\frac{9 \kappa^{2} c^{4}}{8 \pi \tau_{\mathrm{D}} n^{4} R^{6}} \int F_{\mathrm{D}}(\omega) \sigma_{\mathrm{A}}(\omega) \frac{d \omega}{\omega^{4}}
$$

In this expression $\kappa$ is an orientation factor (its detailed form to be given below); $F_{\mathrm{D}}(\omega)$ is the normalized spectrum of donor fluorescence; $\sigma_{\mathrm{A}}(\omega)$ is the absorption cross-section of the acceptor and $\omega$ the optical frequency $(2 \pi v) ; c$ is the speed of light and $\tau_{\mathrm{D}}$ is the radiative lifetime of the donor. It is important to clarify the meaning of $\tau_{\mathrm{D}}$; it represents the rate of radiative decay of the donor from its excited state to the ground state, and is related to the measured fluorescence lifetime $\tau_{\text {fluo }}$ through $\tau_{\text {fluor }}=\eta \tau_{D}$, where $\eta$ is the fluorescence quantum yield. Other sources give an alternative formula:

$$
w=\frac{2 \kappa^{2}}{3} \frac{1}{\tau_{\mathrm{D}}}\left(\frac{R_{0}}{R}\right)^{6}
$$

where $R_{0}$ is called the critical or Förster radius, calculable from the overlap integral of the appropriate fluorescence and absorption spectra; typical values of the Förster radius range over a few nanometres. Apparent in each formula, the inverse sixth power dependence on donor-acceptor separation is a characteristic of dipole-dipole energy transfer. It determines the fact that, provided intermediary donor/acceptor species are suitably positioned, any process resulting in the overall migration of excitation over distances beyond the Förster radius will mostly operate through a series of short hops rather than one long hop (but see below regarding the super-exchange mechanism and bridging effects). The $\kappa$ factor in the rate equation depends on the orientations of the donor and acceptor, both with respect to each other and with respect to the donor-acceptor displacement unit vector $\mathbf{R}$ :

$$
\kappa=\left(\hat{\mu}_{D} \cdot \hat{\mu}_{A}\right)-3\left(\hat{R} \cdot \hat{\mu}_{D}\right)\left(\hat{R} \cdot \hat{\mu}_{A}\right) .
$$


Unfavourable orientations can reduce the rate of energy transfer to zero - others, including many of those found in Nature, will optimize the transfer rate. The angular disposition of chromophores is therefore a very important facet of energy transfer, and one that invites careful consideration in the design of energy harvesting materials.

The dipole-dipole interaction which leads to the above results is in certain cases not applicable, as for example if either the donor or acceptor transition is electric dipole (E1)-forbidden or exceptionally weak. Then, the coupling can involve the electric quadrupole moment (E2), higher electric multipoles (En) or even magnetic multipoles (Mn), in each case leading to an orientation and distance dependence of a different form. In the most common case of predominantly electric coupling, then if (En) and (Em) are the leading non-zero moments of the donor and acceptor, the distance dependence takes the form $R^{-2(n+m+1)}$. [12,13] Observations of energy transfer associated with dipole-forbidden interactions often reveal a degree of efficiency that precludes explanation simply on the basis of higher-order multipole coupling, however. In 1953 Dexter offered a theory to account for such observations, [14] based on the premise that when the electron distributions of the donor and acceptor are close enough to overlap strongly, the energy of electronic excitation can pass directly to the acceptor, essentially channeled by the overlapping electron clouds. The Dexter exchange mechanism is exponentially dependent on the distance between donor and acceptor, reflecting a typical asymptote for the wavefunctions of the molecular orbitals; as such it comes into play only at very short distances where the electron clouds overlap strongly. It is not always appreciated that the Dexter mechanism can operate over such short distances both for dipole-forbidden and also dipole-allowed transitions; for an account of how the Dexter and Förster mechanisms seamlessly merge for the dipole-allowed case, see Ref. [15]. When donor and acceptor chromophores are electronically distinct, however, one should account for dipole-forbidden transitions in terms of higher multipoles.

It is known that the dielectric properties of the medium within which energy transfer takes place (the protein matrix in photosynthetic systems) exercises a considerable influence on the transfer efficiency, beyond the simple refractive dependence exhibited in the Förster formula. [16] Where electronically prominent chromophore species intervene between donor and acceptor groups, the detailed understanding of such influence has to be sought at the molecular level. For example, one obvious factor in determining the efficiency of excitation transfer is possible mediation of the transfer through the electronic involvement of a bridge or other suitable placed species. Work on rigidly linked bichromophore molecules has given conclusive evidence that, under certain conditions, a through-bond super-exchange interaction could be the dominant feature of an energy transport process. [17] A key piece of work in connection with photosynthetic systems, for example, established that ancillary carotenoid pigments can help mediate B800-B850 coupling in LH2. [18] A recent analysis based on molecular QED has re-examined a number of the broader issues associated with the electronic influence of a third body on resonance energy transfer, showing how it can be tailored to effectively 'switch on' the energy transfer process under circumstances where it is normally forbidden due to orientational effects or on symmetry grounds. [19]

Turning to the case of closely neighbouring, electronically distinct but identical chromophores, striking differences are seen to emerge as a result of the formation of excitons through the quantum interference of states, a direct result of the stronger coupling. [20] At simplest, the excited states of any isolated pair (comprising a strongly coupled donor and acceptor) can mix and form a joint excited state split by twice the coupling energy. With such excitonic states one can no longer specify the molecular location of the electronic excitation, as it is delocalised. By extension, in lightharvesting complexes comprising large numbers of equivalent chromophores, excitons can spread over several equivalent donor/acceptor species and be associated with numerous, closely separated energy levels. Indeed, the separation between the lowest levels affords a useful means of gauging the extent of exciton delocalisation. [21] Although any local disorder can substantially compromise the extent of excitonic delocalisation and also any superexchange coupling, [22] a surprising finding in connection with B800-B850 coupling was that site disorder can be responsible for a more rapid transfer of excitation than would occur in its absence. [23] In multi-chromophore arrays the very multiplicity of the associated exciton splitting enhances spectral overlap and thereby accelerates energy transfer exhibit, leading to coherent energy transfer. Thus it is that in natural light-harvesting complexes, the ring structures, which provide symmetry in the pigment arrays, enhance absorption and lead to those arrays as a whole acting as nanoscale energy traps. The mechanistic interpretations of many bacterial and other photosynthetic systems are based on this premise. [24-26] For a comprehensive review of the role and characterization of excitons in photosynthesis see Ref. [27]. 


\section{DENDRIMERIC SYSTEMS}

Following several decades in which the science of energy harvesting materials was largely embedded in the development of photovoltaic cells, the arrival of dendrimeric macromolecules heralded a new opportunity for achieving more closely biomimetic light harvesting. [28,29] Dendrimers are multi-branched structures with essentially fractal geometry and a large number of chemically similar chromophores amongst which energy can transfer; as such they are also known as functional cascade molecules. [30] In the typical example shown in Fig. 2, some of the features which are also common to natural photosynthetic systems are immediately apparent; principally the circularly symmetric ringlike array of chromophores about a central excitation trap. However not only these structural motifs are important; it is also highly significant that the chromophore units are electronically distinct, here a consequence of the alternate $1,3,5-$ substituent linkage pattern on each benzene ring. In the example shown the ether linkage has the effect of electronically isolating the phenyl chromophores; ethynyl linkages are also commonly employed in such dendrimers to similar effect, as detailed theory [28,31] and spectroscopic evidence [32] has shown. As a result there is negligible through-bond coupling between the chromophore units, and the transfer of excitation between them essentially takes the form of dipole coupling. In any dendrimer the synthetic route gives successive generations, each expanded by the peripheral addition of further functional groups. Clearly the central unit, even when of chemically identical form to the dendritic

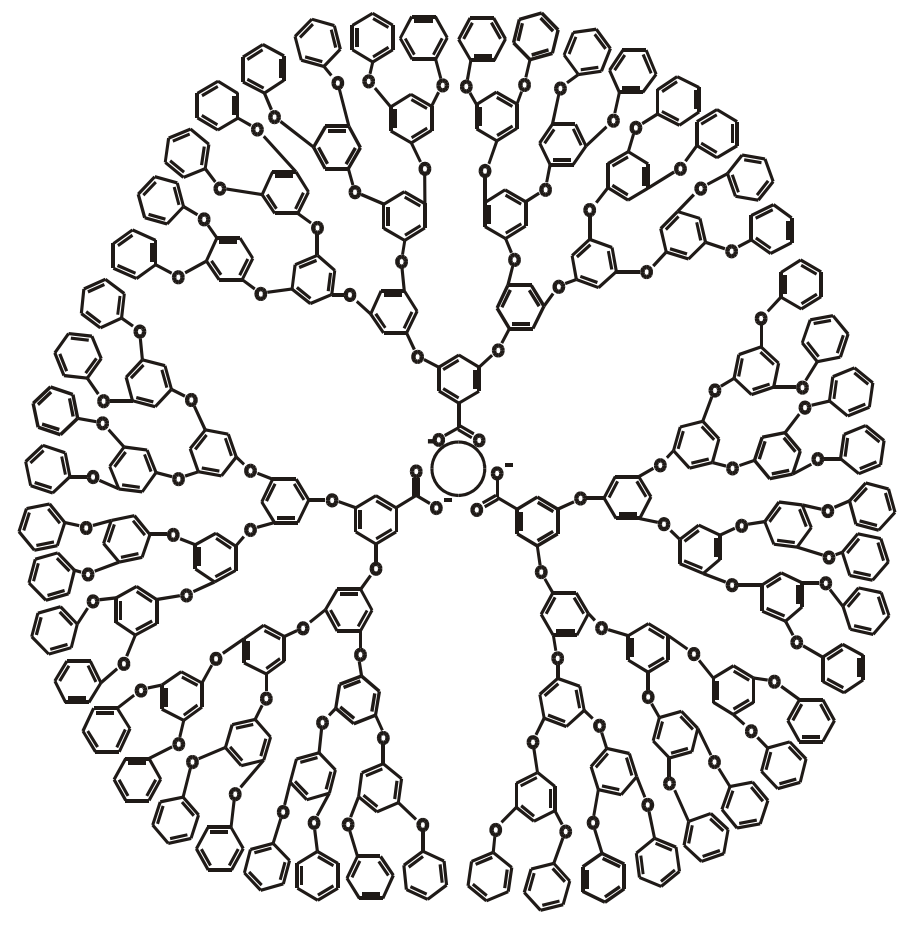

Fig. 2: Polyphenylether dendrimer with a photoactive trap addenda, differs in its electronic environment from those chromophores in the dendrite arms; equally, the peripheral chromophores are in a still different environment. This, too, can be played to advantage; in ideal cases the associated shifts in the corresponding absorption and fluorescence spectra can foster exactly the kind of spectroscopic or energy gradient observed in natural systems. [33] This energy funnel feature here represents the combination of structural and spectroscopic elements which together direct energy towards the core. The latter acts as the trap. The efficient, unidirectional transfer of energy within a dendrimeric frame towards a single core chromophore was first reported only as recently as 1994. [34]

Much current work in this area is directed towards the use of photoactive dendrimers as model systems - not only for photosynthetic apparatus, but also as light-emitting diodes, fluorescent sensors, frequency conversion materials and other photonic devices. Future advances are expected to lead towards more directly biomimetic energy harvesting systems. One of the clear objectives is to achieve through funneling and trapping the kind of energy pooling that characterizes the biological systems, where the energy of more than one photon is deposited in the trap - a subject we shall return to below. Given sufficient levels of input intensity, the possibility arises of having two or more chromophores simultaneously excited (most probably in different dendrite arms) and this suggests the possible exploitation of optical nonlinearity. There is already some evidence to suggest the operation of such mechanisms in photoresponsive dendrimers, particularly those based on an azobenzene core with a pair of benzyl aryl ether dendrites. Here the energy required to effect the observed cis-trans isomerization of the core appears to be only consistent with the pooling of excitation from two of the chromophores. [35,36] Schemes of this kind have for some time been very successfully implemented in rare earth crystals, and it is instructive to examine the principles that operate in that once again very different area of materials science - particularly since the excitation of lanthanide ions is faster and does not effect structural change. In biosystems the end product of energy harvesting is designedly synthetic, leading to the growth of cells and a concomitant change of shape; in artificial light-harvesting materials, retention of structure is more desirable. 
Although not strictly dendrimeric, other light-harvesting materials with analogous structural motifs have also been investigated. Closer to Nature, multi-porphyrin systems in particular appear to offer considerable promise. [37] Here for example it has been shown that excitation transfer between a zinc porphyrin acting as donor and its free base counterpart as acceptor can be significantly enhanced by exploiting suitable bridging chromophores. [38] As with the dendrimers, these structures are designed to obviate any transfer through charge conjugation or wavefunction overlap between donor and acceptor species. Other recent work has forged a synthetic and conceptual link in this area, through the synthesis and characterization of multi-porphyrin arrays based on a phenylethynyl proto-dendrimeric framework. [39] A striking success is the artificial photosynthetic antenna-reaction center complex comprising four zinc tetraarylporphyrins covalently linked, through phenylethynyl dendrites, to a free base porphyrin-fullerene 'reaction center', the charge-separated excited state of which is generated with a quantum yield of 0.7 , based on the light absorbed by the zinc porphyrin antenna. [40]

\section{RARE EARTH MATERIALS}

Since Kaiser and Garrett's pioneering work on two-photon absorption, [41] observations of blue-shifted fluorescence have been widely used as a marker and measure of nonlinear excitation. In rare-earth doped crystals, as in other complex multichromophore systems, resonance energy transfer commonly conveys excitation away from the sites of initial photoabsorption, and a possible intermediary role for this type of energy transfer in connection with two-photon fluorescence has been recognised. [42] Nonetheless, two-photon fluorescence resonance energy transfer is not the only process which can indirectly lead to blue-shifted fluorescence in rare earth materials at high levels of excitation. [43-45] There are other competing mechanisms for such observations, each involving energy transfer between three chromophore/fluorophore sites, with two acting as donors and one as acceptor. For an overview of three-body in the context of other photophysical processes involving lanthanides the reader is referred to a review by Auzel. [46] Twindonor mechanisms should be prominent in strongly pumped lanthanide materials, given suitable conditions. [47-49] The reason that rare earth ions are so favoured in this connection is that their $f-f$ transitions are associated with a redistribution of charge sufficiently close to the nucleus that the ionic environment exercises relatively little influence; as a consequence, their absorption and fluorescence spectra contain highly discrete and well-resolved lines that immediately characterize the lanthanide. [50-52] For this reason, such ions are also widely employed as markers in FRET measurements on proteins, and in bioimaging applications for example.

The processes of interest can occur in any system containing a reasonably high density of chromophores. When the laser irradiation of such a system produces a significant excited state population of one species, fluorescence can be exhibited at a wavelength shorter than usual as a result of energy pooling, either in the same or in a chemically different species. In detail, the process of interest here entails resonance energy transfer from initially excited fluorophore sites $A$ and $B$ (which may, but need not, be chemically identical to $A$ ), to other fluorophores $C$. As illustrated in Fig. 3 the sequence $A B C$ signifies that in each transfer event the excitation energy of $A$ is routed to $C$ via $B$, at which intermediate point the additional excitation energy of $B$ is acquired. The sum energy thereby delivered to $C$ accomplishes the excitation of $C$, whose ensuing fluorescent decay produces the observed blue-shifted radiation - the overall process known as up-conversion. The pooling of energy at $C$, which should not be thought of as a two-step process since energy need not be conserved before its completion, has been designated an accretive mechanism, having due regard to the nature of the intermediary role of $B$. $[48,49]$

An alternative process, by means of which energy is deposited at $C$ directly from the two donors, is termed cooperative; [47,49] both are illustrated in Fig. 4. To observe such energy pooling, in any system accommodating donor and acceptor species appropriate for the laser input, it is necessary to furnish the necessary levels of donor excitation. The specific requirement here is for pairs of donors, $A$ and $B$, to be excited through conventional single-photon absorption - so that during a certain period, they are simultaneously in excited states. In the simplest practical case the donor species are identical, and excited by the same input radiation. Detailed calculations have shown that two-site single-photon absorption is not intrinsically less probable than the more familiar single-site process of two-photon absorption - in general, pulsed

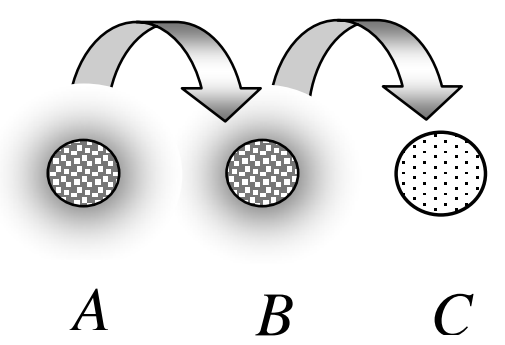

Fig.3: Accretive energy transfer 


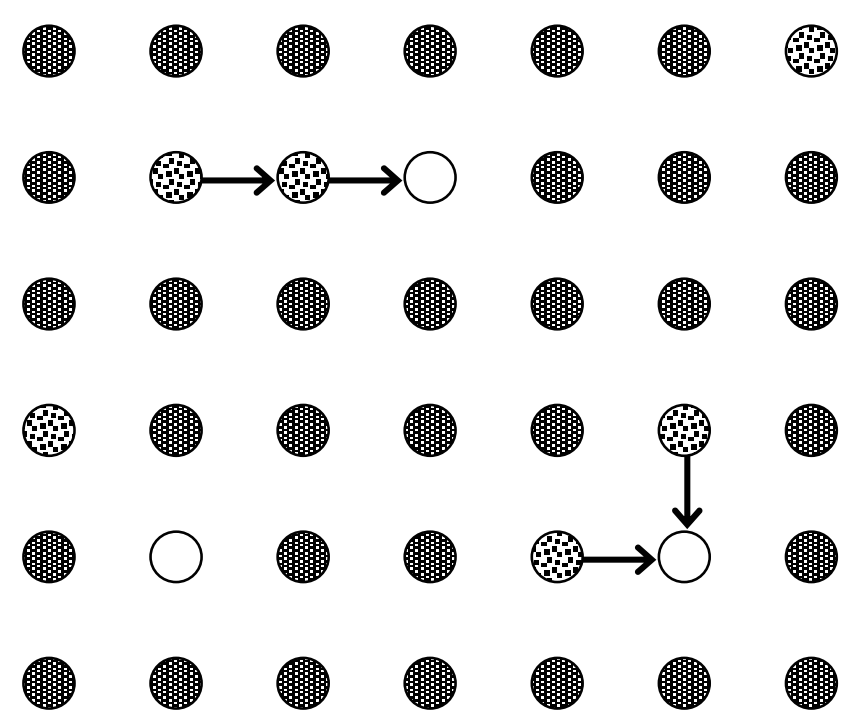

Fig.4: Accretive (upper left) and cooperative (lower right) energy pooling. Dotted circles - excited donors: open circles - acceptors: dark circles - ground species. laser irradiation can readily afford the means of establishing the necessary level of donor excitation for both cooperative and accretive energy pooling. In the optically primed system, the relative number densities of excited donors and acceptors is a significant determinant of mechanism, coupled with the different optical selection rules that operate for each mechanism. Specifically, the cooperative mechanism requires the acceptor excitation to satisfy the same selection rules as two-photon absorption.

Examples of twin-donor energy pooling in upconversion are rife, and not necessarily limited to systems containing more than one species of rare earth ion. Occurrence of the process has for example been identified in systems involving concerted action amongst three electronically excited $\operatorname{Pr}^{3+}$ ions, in which two ions decay and the third is further excited through the acquisition of their combined energy. [53,54]. An iso-ionic process also accounts for both green and red zmissions from $\mathrm{Er}^{3+}$ embedded in fluorite-type crystals; see Ref. 55.

\section{MODELING ADVANCED MATERIALS}

Implementing in dendrimeric and other more closely biomimetic light-harvesting arrays some of the design principles that work for rare earths offers enormous scope for future developments. In this connection theoretical work is progressing hand in hand with current synthetic efforts, each informing the other; in both areas the difficulties are considerable, yet good progress is being made. The theory alone accommodates principles ranging from quantum mechanics to non-equilibrium thermodynamics. [56] One part of the complexity arises from the untrammeled scope for

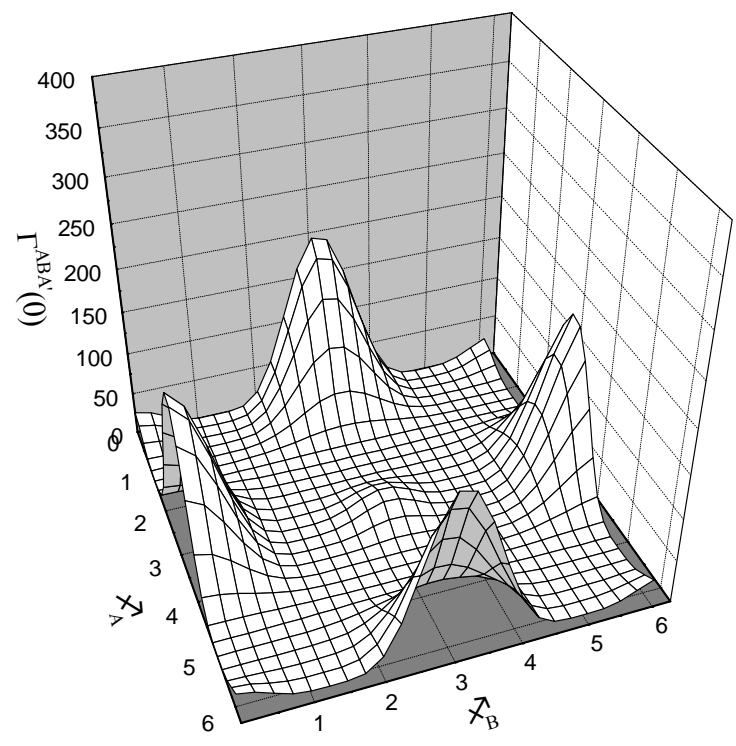

Fig.5: Variation of cooperative energy transfer rate with chromophore geometry (see text). novel molecular architecture. For natural systems, progressive revelation of the light-harvesting structures has enabled theory to home in with increasing focus on the interplay of specifically relevant electronic properties. However where artificial systems are concerned, there is a carte blanche.

Considering for example the intricacies which arise in connection with the orientational factor even for the simplest donor-acceptor system, it is not surprising to find that there is a rich ground for exploration where multi-chromophore arrays are concerned; indeed, for their full analysis, multidimensional optimization routines are required. By way of illustration, Fig. 5 shows a plot of the variation of efficiency for the cooperative transfer of energy to a central trap in a linear twin-donor system, as a function of the angles made by the transition dipole moments of the two donors, and that of the acceptor, relative to an axis on which all three chromophores lie. For the calculation of this three-dimensional plot it is assumed that all three transition moments are coplanar and that both donor moments are collinear - and the results applies just for one of the two contributing mechanisms. [57] For any real system the accretive mechanism also invites appraisal by similar means. 
Some recent theory has highlighted the need to simplify the conceptualization of multi-chromophore energy pooling through the application of a new interaction-pair terminology, not least to obviate the conceptual and even semantic problems which can often arise in addressing multi-center interactions. [58] In connection with triple-donor pooling, for example, a depiction as in Fig. 6 of the overall light-harvesting scheme for a model dendrimer illustrates that four mechanisms with varying degrees of accretive and cooperative character apply, yet the diagram fails to convey the significant fact that not any one mechanism operates alone, unless the chromophore system is such that the selection rules or the geometric arrangement preclude the others. Detailed QED calculation shows that the rate of energy harvesting has contributions not only associated with each of the routes individually, but also from their quantum interference, which may signify either an enhancement or a reduction in the overall harvesting efficiency. Moreover for a multi-chromophore structure the calculation of interference terms has to accommodate all possible chromophores as intermediaries.
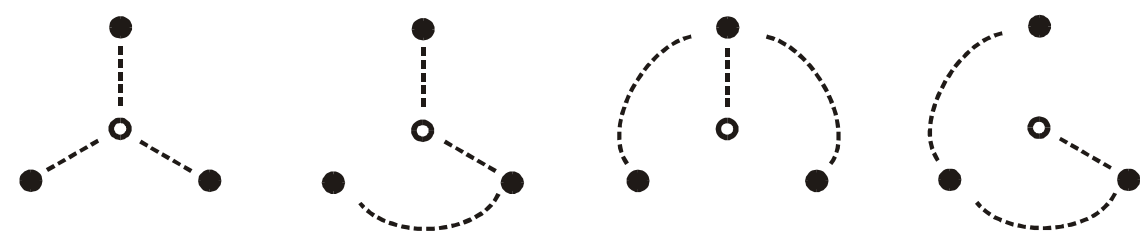

Fig. 6: Four mechanistic pathways for triple-donor energy pooling in a model dendrimer

One other quantum feature that arises here concerns the time-ordering of the elementary interactions. The above figure designedly omits any arrowheads suggesting directions or orderings for the virtual photon couplings from which each mechanistic pathway is constructed, because all temporally distinct orderings must in fact be accommodated in the theory, in order to correctly calculate the form of the electronic tensors for the chromophores involved. This is a simple consequence of the time-energy uncertainty principle; although its application to such systems is counter-intuitive, its disregard would certainly engender significant error.

\section{CONCLUSION}

The unparalleled interdisciplinarity manifest in the science of energy harvesting materials offers scope to accommodate insights and also to exploit design strategies from a host of different subject areas. In this brief review attention has focused on the principles and mechanisms of biological photosynthesis, photoactive dendrimers and rare earth optoelectronic materials. Future efforts will undoubtedly lead to the devising of more closely biomimetic analogues of natural photosynthetic light-harvesting arrays; other promising avenues for research suggest the further development of materials that can exploit novel forms of optical nonlinearity for the harvesting of energy under more intense irradiation. Such behavior is associated with the direct pooling of excitation energy, by means of which secondary acceptors undergo transitions to states whose energy equates to that of two or more input photons, subject to decay losses. Observations of this kind have already been made on a variety of materials, ranging from photoactive dyes, through fullerene derivatives, to lanthanide-doped crystals. Recently developed theory has further established the underlying principles and links between the modes of operation of these systems. Key factors include the chromophore layout and geometry; also the electronic structure and optical selection rules. The fundamentally quantum mechanical nature of the coupling is also manifest in the detailed form of the associated response tensors. Properly assessing the interplay of these factors in photoactive materials design will prove crucial for the design of new materials for operation with laser input.

\section{ACKNOWLEDGEMENTS}

I am pleased to acknowledge many useful discussions with Dr Robert D. Jenkins (UEA), who also produced many of the graphics. Work conducted in the QED group at the University of East Anglia is funded by the Engineering and Physical Sciences Research Council. 


\section{REFERENCES}

1. R. van Grondelle and O. J. G. Somsen, pp. 366-398 in Resonance Energy Transfer, ed. D. L. Andrews and A. A. Demidov, Wiley, New York, 1999.

2. X. Hu and K. Schulten, Phys. Today 50 (8), 28 (1997).

3. K. Schulten, pp. 227-253 in Simplicity and Complexity in Proteins and Nucleic Acids, ed. H. Frauenfelder, J. Deisenhofer and P. G. Wolynes, Dahlem University Press, Berlin, 1999.

4. Th. Förster, pp. 93-137 in Modern Quantum Chemistry, ed. O. Sinanoglu, Academic Press, New York. 1965.

5. V. M. Agranovich and M. D. Galanin, Electronic Excitation Energy Transfer in Condensed Matter, Elsevier/NorthHolland, Amsterdam, 1982.

6. B. W. van der Meer, J. Coker III, and S.-Y.S. Chen, Resonance Energy Transfer: Theory and Data, VCH, New York, 1982.

7. Th. Förster, Annalen der Physik 2, 551948.

8. D. L. Andrews, Chem. Phys. 135, 195 (1989).

9. D. L. Andrews and G. Juzeliūnas, J. Chem. Phys. 96, 6606 (1992).

10. D. L. Andrews and P. Allcock, Chem. Soc. Rev. 24, 259 (1995).

11. G. Juzeliūnas and D. L. Andrews, Adv. Chem. Phys. 112, 357 (2000).

12. G. D. Scholes, A. H. A. Clayton and K. P. Ghiggino, J. Chem. Phys. 97, 7405 (1992).

13. G. D. Scholes and D. L. Andrews, J. Chem. Phys. 107, 5374 (1997).

14. D. L. Dexter, 1953, J. Chem. Phys. 21, 836 (1953).

15. G. D. Scholes and K. P. Ghiggino, J. Phys. Chem. 98, 4580 (1994).

16. C.-P. Hsu, G. R. Fleming, M. Head-Gordon and T. Head-Gordon, J. Chem. Phys. 114, 3065 (2001).

17. K. P. Ghiggino, E. K. L. Yeow, D. J. Haines, G. D. Scholes and T. A. Smith, J. Photochem. Photobiol. A: Chemistry 102, 81 (1996).

18. B. P. Krueger, G. D. Scholes, I. R. Gould and G. R. Fleming, PhysChemComm, 8 (1999).

19. G. J. Daniels and D. L. Andrews, J. Chem. Phys. 116, 6701 (2002).

20. D. P. Craig and T. Thirunamachandran, Chem. Phys. 167, 229 (1992).

21. L. D. Bakalis and J. Knoester, J. Lumin. 87-89, 66 (2000).

22. E. K. L. Yeow and K. P. Ghiggino, J. Phys. Chem. A 104, 5825 (2000).

23. G. D. Scholes and G. R. Fleming, J. Phys. Chem. B 104, 1854 (2000).

24. J. R. Durrant, D. R. Klug, S. L. S. Kwa, R. Van Grondelle, G. Porter and J. P. Dekker, Proc. Nat. Acad. Sci., 92, 4798 (1995).

25. S. Gnanakaran, R. Haran, R. Kumble and R. M. Hochstrasser, pp. 308-365 in Resonance Energy Transfer, ed. D. L. Andrews and A. A. Demidov, Wiley, New York, 1999.

26. S. Savikhin, D. R. Buck and W. S. Struve, pp. 399-434 in Resonance Energy Transfer, ed. D. L. Andrews and A. A. Demidov, Wiley, New York, 1999.

27. H. van Amerongen, L. Valkunas and R. van Grondelle, Photosynthetic Excitons, World Scientific, New Jersey, 2000.

28. T. Minami, S. Tretiak, V. Chernyak and S. Mukamel, J. Lumin. 87-89, 115 (2000).

29. A. Adronov and J. M. J. Fréchet, Chem. Commun. 1701 (2000).

30. A. Archut and G. Vögtle, Chem. Soc. Rev. 27, 233 (1998).

31. S. Tretiak, V. Chernyak and S. Mukamel, J. Phys. B 102, 3310 (1998).

32. R. Kopelman, M. Shortreed, Z.-Y. Shi, W. Tan, Z. Xu, J. S. Moore, A. Bar-Haim and J. Klafter, Phys. Rev. Letts 78, 1239 (1997).

33. C. Devadoss, P. Bharathi and J. S. Moore, J. Am. Chem. Soc. 118, 9635 (1996).

34. Z. F. Xu and J. S. Moore, Acta Polym. 45, 83 (1994).

35. D. M. Junge and D. V. McGrath, Chem. Commun. 857 (1997).

36. D.-L. Jiang and T. Aida, Nature 388, 454 (1997).

37. P. G. Van Patten, A. P. Shreve, J. S. Lindsey and R. J. Donohoe, J. Phys. Chem. B 102, 4209 (1998).

38. K. Kilså, J. Kajanus, J. Mårtensson and B. Albinsson, J. Phys. Chem. B 103, 7329 (1999).

39. P. Brodard, S. Matzinger, E. Vauthey, O. Mongin, C. Papamicaël and A. Gossauer, J. Phys. Chem. A 103, 5838 (1999).

40. D. Kuciauskas, P. A. Liddell, S. Lin, T. E. Johnson, S. J. Weghorn, J. S. Lindsey, A. L. Moore, T. A. Moore and D. Gust, J. Am. Chem. Soc. 121, 8604 (1999). 
41. W. Kaiser and C. G. B. Garrett, Phys. Rev. Letts 7, 229 (1961).

42. P. Allcock and D. L. Andrews, J. Chem. Phys. 108, 3089 (1998).

43. A. Saïssy, B. Dussardier, G. Maze, G. Monnom, S.A. Wade, Opt. Fibre Technol. 2, 249 (1996).

44. M. Chua and P. A. Tanner, J. Lumin. 66\&67, 203 (1996).

45. X. Zhang, J.-P. Jouart and G. Mary, J. Phys: Cond. Matter 10, 493 (1998).

46. F. Auzel, J. Lumin, 45, 341 (1990).

47. R. D. Jenkins and D. L. Andrews, Chem. Phys. Letts 301, 235 (1999).

48. D. L. Andrews, J. Raman Spectrosc. 31, 791 (2000).

49. D. L. Andrews and R. D. Jenkins, J. Chem. Phys. 114, 1089 (2001).

50. B. G. Wybourne, Spectroscopic Properties of Rare Earths, Interscience, New York, 1965.

51. G. H. Dieke, Spectra and Energy Levels of Rare Earth Ions in Crystals, Wiley, New York, 1968.

52. V. V. Ovsyankin, pp.343-480 in Spectroscopy of Solids Containing Rare Earth Ions, ed. A. A. Kaplyanskii and R. M. Macfarlane, Elsevier, Amsterdam, 1987.

53. L.-S. Lee, S. C. Rand, and A. L. Schawlow, Phys. Rev. B 29, 6901 (1984).

54. A. Lezama, M. Oriá, J. R. Rios Leite, and Cid B. De Araújo, Phys. Rev. B 32, 7139 (1985).

55. J. P. Jouart and G. Mary. J. Lumin. 46, 39 (1990).

56. T. Markvart, Prog. Quantum Electron. 24, 107 (2000).

57. R. D. Jenkins and D. L. Andrews, Phys. Chem. Chem. Phys. 2, 2837 (2000).

58. R. D. Jenkins and D. L. Andrews, J. Chem. Phys. 116, 6713 (2002). 